\title{
Underlying Causes and Nursing Care in Infections Associated with Peripheral Venous Catheterization in Children
}

\author{
Aysegul Simsek \\ School: Istanbul University Cerrahpasa Institute of Graduate Education, Istanbul, Turkey, \\ Work: Istinye University, Istanbul, Turkey \\ E-mail: aysegul.simsek@istinye.edu.tr \\ Suzan Yildiz \\ Istanbul University Cerrahpasa Istanbul, Turkey \\ E-mail: suzyil58@gmail.com
}

\begin{abstract}
Peripheral intravenous catheterization is one of the main applications in the treatment of diseases. A correct catheterization application helps treatment. A mistake or inability during catheterization process causes many complications, especially catheter-related infections. Therefore, an effective catheterization process, especially in the childhood group, requires a trained and equipped nursing care.
\end{abstract}

Index Terms: Alcohol, antiseptic, catheter related infections, children, chlorhexidine gluconate, peripheral intravenous catheter

\section{Special Issue of Health Sciences}

DOI: $10.7176 / \mathrm{JSTR} / 6-03-38$

\section{INTRODUCTION}

Intravenous therapy, which is one of the cornerstones of healthcare from past to present, is the most commonly used route among nursing practices (O'Grady et al, 2011; Pujol et al, 2007; Rai et al, 2019). Peripheral venous catheterization (PVC) is administered in patients admitted to hospitals or medical centers for inpatient or outpatient treatment and evaluation or for intravenous treatments of the patients (Hadaway, 2012; Özyazıcıŏglu \& Arıkan, 2007). PVC is commonly used in inpatients during processes such as providing hydration, parenteral nutrition, hemodynamic monitoring, antibiotic and drug treatment, chemotherapy, transfusion of blood or blood products, liquid and electrolyte replacement (Özyazıcı̆ğlu \& Arıkan 2007; Camacho-Ortiz \& Roman-Mancha, 2016; Uslusoy, 2006; Rai et al, 2019; Choudhury et al, 2019).

\section{HISTORY}

Throughout human history, various methods have been tried for diagnosis and treatment of diseases and inserting a catheter into a vein for intravenous treatments has been the most frequently used tool (Biçer, 2017; Paşalığlu, 2012). The first known applications date back to the 15th century and the first intravenous administration is the blood transfusion from two healthy people to Pope Innocent VIII performed in Vatican. However, since the blood groups were unknown, this transfusion has resulted with death. In the 17th century, a venous drug substance (opium) was administered to a dog via a hollow hair after discovery of blood circulation (Karagözoğlu, 2001; Cihan Erdoğan, 2014). Following these developments, the first intravenous catheter was also produced in the 17 th century.

Again, in the same century, the first blood transfusion was attempted from an animal to a human and was resulted by death of the subject. These attempts were prohibited by governments due to the death of subjects and the lack of information accompanying inadequate technology.

Blood transfusions from person to person initiated again in the 19th century in England in order to prevent deaths caused by postpartum hemorrhage. The first intravenous drug administration in humans was water and salt replacement to a patient with cholera diagnosis (Cihan Erdoğan, 2014). Intravenous catheters were made of steel until the 20th century, and the first plastic catheters were used by Mayer in 1945. After this development, advances in intravenous therapy have accelerated (Şimşek, 2012). While 
intravenous treatments were carried out only by physicians until the 20th century, a nurse at Massachusetts General Hospital was trained for intravenous treatments in 1940, and after this advancement, it was established that nurses can also take responsibility for these practices (Biçer, 2017).

\section{INCIDENCES}

Since PVC is the most commonly used application in health system in the world, provides great benefit in all age groups (Aktaş et al, 2010). However, in addition to its benefits, it also increases the incidence of mortality and morbidity because of the complications caused by factors both related to the catheter, the treatment administered and the patient (Camacho-Ortiz \& Roman-Mancha, 2016; Aktaş et al, 2010; Pujol et al, 2007). Catheter-related infections (CRI) have a large proportion among these complications and they might be the most important one. CRIs are defined with local inflammation findings such as redness around the catheter and increased heat, as well as systemic findings such as fever, chills, and changes in blood analysis (Serane \& Kothendaraman, 2016). CRI is one of the most three common hospital related infections. CRI incidence was reported as $0.7-17 \%$ and the incidence of PVC among them was reported as $0.2-0.5 \%$ (Yasuda et al, 2017; CDC, 2018). Considering the data obtained from hospital records, CRIs were classified as catheter-related blood stream infections in 13.9\% $(\mathrm{n}=201)$, catheter entry site infections in $6.4 \%$ and colonizations in $27.3 \%$ of the patients admitted in a one-year period (Aktaş et al, 2010).

\section{RISK FACTORS AND COMPLICATIONS}

The most commonly used PVC is one of the minor surgical procedures that require practive skills and causes various complications if not done properly. PVC-related catheter complications are sorted as pain, occlusion, cellulitis, sepsis, thrombophlebitis, infiltration / extravasation, air embolism, nerve tendon damage, subcutaneous necrosis, circulatory loading, accidental dislocation or stealing of the catheter, hematoma and CRIs (Hadaway, 2012; Zhang et al, 2016 Rai et al, 2019; Karagözoğlu 2001; Wu et al, 2017; Aktaş et al, 2011; Rundjan, 2011; Camacho-Ortiz \& Roman-Mancha, 2016).

The development of complications depends on factors such as age, gender, weight, region selection, material and length of the catheter that will be used, the type and osmolarity of the fluid or drug that will be applied, presence of chronic diseases, dressing material that will be used, the level of knowledge and experience of the person that will install the catheter, and the aseptic technique compliance during administration. (Cihan Erdoğan, 2014). Intravenous catheter complications cause prolonged hospitalization, exposure to extra examination and treatment procedures, stress, economical losses and additional workload for healthcare stuff (Timsit et al, 2011). Complications could be avoided by careful evaluation and monitoring of the patient and proper administration (Cihan Erdoğan, 2014; Rai et al, 2019; Timsit et al, 2011).

There are many trials about PVC related infect, and when these studies are evaluated, it was determined that most of them have been performed in adults and trials in pediatric population were very less (Serane \& Kothendaraman, 2016). In addition to age-related factors for pediatric patients; risk factors for infection occurrence such as body weight, coexisting chronic disease, medical diagnosis, characteristics of the catheter used and the drug administered, presence of interconnection are different from adults and a separate discussion is needed for CRI in children (Timsit et al, 2011).

\section{DIAGNOSIS}

Clinical findings (evaluation of local and systemic findings) and microbiological analysis are important for diagnosis (Öztürk, 2000). The patient should be observed systematically and locally in terms of CRI findings, and vital findings should be measured in regular intervals.

However, since the exact diagnosis of CRI is established by microbiological methods, a certain period of time is required to determine the agent and establish a diagnosis (Öztürk, 2000). In microbiological diagnosis, the swab culture taken from the catheter and the catheter entry should be examined (Öztürk, 2000). Diagnosis of CRI is based on the presence of positive culture results in venous blood analysis and samples taken from the catheter or catheter entry site (Zhang et al, 2016).

In the presence or suspicion of a systemic infection, blood culture should be taken, and the catheter should be removed (Öztürk, 2000). If necessary, the drawn catheter tip should be sent for culture (Zhang et al, 2016; Öztürk, 2000).

In the presence of local infection signs (swelling, redness, heat rise, and pain), the catheter should be removed (O'Grady et al, 2011). If purulent discharge is present, sample should be sent for culture. 


\section{TREATMENT}

The treatment varies according to the type of infection (local, systemic), agent microorganism, catheter type and status of the host (immune status, underlying disease) (Öztürk, 2000). In cases with local infection and the infection source is the catheter, it is adequate to remove the catheter (O'Grady et al, 2011). Antibiotherapy should be initiated due to the culture result obtained from the catheter and the catheter entry site. Routine antibiotherapy administration should be avoided (O'Grady et al, 2011; Öztürk, 2000). Antipyretics should be added to the treatment when high fever is present. The patient should be observed in terms of infection parameters and vital findings should be recorded by measurements in regular intervals.

\section{PREVENTION AND NURSING CARE}

Participation of nurses in IV examination and treatment practices initiated after the mid-twentieth century, and subsequently, working groups were constituted to determine how nurses would safely and correctly carry out these practices, and reports were published (Biçer, 2017). Three main headings including the rules and precautions that nurses should follow during IV treatment, asepsis, safety of patients and healthcare professional was stated in these reports (Biçer, 2017; HIDER, 2019). According to these titles, the following protection precautions should be taken:

Education: Because catheterization and intravenous treatment is one of the nursing practices, nurses should be trained about these topics initiating with undergraduate education (Özyazıcıoğlu \& Arıkan 2007; Yasuda et al, 2017; O'Grady et al, 2011; Zhang et al, 2016).

Intravenous catheterization teams: Recently, in order to carry out IV catheterization and treatment process in a healthy way, it is necessary to primarily constitute practice-oriented teams (Biçer, 2017). PVC teams should be should take part in every stage of the PVC process and be fully equipped (Biçer, 2017). It was reported in trials that the rate of catheter infection occurrence decreases by an equipped team (Zhang et al, 2016; O’Grady et al, 2011).

Number of efforts: The PVC procedure should not be tried more than twice by the practitioner, and it should be re-evaluated and attempted by another nurse, physician or anesthesiologist after two unsuccessful attempts (Cihan Erdoğan, 2014).

Prevention packages: Catheterization packages (bundles) containing standardized maximum barrier and asepsis precautions should be used for management of catheterization process to prevent PVC-related infections (Timsit et al, 2011; O'Grady et al, 2011). In these packages, rules such as hand washing, healtcare professional training, patient privacy, material preparation, skin antisepsis, providence of barrier methods, daily evaluation of catheters and removal of the catheter when it is not needed are reported (O'Grady et al, 2011, CDC, 2018; Avc1, 2015; Özyazıcığlu \& Arıkan, 2007; Timsit et al, 2011; Boyd et al, 2011).

Catheter care and observation: Every patient that an intravenous catheter was applied should be checked daily by inspection and palpation methods for signs and findings of local and systemic infection (O'Grady et al, 2011; Timsit et al, 2011). The dressing should be changed if it is dirty and wet (Cihan Erdoğan, 2014).

Patient education: Children and their parents should be taught to report any abnormality in catheter site or a general disturbance to nurses (O’Grady et al, 2011; Özyazıcıŏlu \& Arıkan, 2007).

Catheter existance period: Unless there is a complication such as phlebitis, PVC should be remained in its place until completion of treatment in pediatric patients (HIDER, 2019).

Catheter insertion site: Scalp, hand and foot veins should be preferred primarily, instead of legs, arms and antecubital regions for cannula site selection in children (O'Grady et al, 2011; HIDER, 2019; Cihan Erdoğan, 2014).

Hand Hygiene and Aseptic Technique: Because most of the agents responsible for CRI are originated from skin flora, it is important to follow hang hygiene ruled and asepsis techniques (O'Grady et al, 2011; Çaylan, 2007; Zhang et al, 2016; Özyazıcıŏlu \& Arıkan, 2007). 
In combination with a non-touch technique for PVC, it is adequate to use disposable clean gloves for prevention of CRI (Zhang et al, 2016; O'Grady et al, 2011; Choudhury et al, 2019). Unless the aseptic technique is preserved, palpation of the joint area should be avoided after antiseptic application. (O’Grady et al, 2011).

Skin antisepsis: The cannula entry site should be wiped with a highly efficient antiseptic solution that is safe for children before inserting the catheter (HIDER, 2019). It was reported in a study that as a consequence of providing skin antisepsis and hand hygiene, the rate of CRI is decreased from $11.7 \%$ to $1.1 \%$ (Rundjan, 2011), and similar studies confirmed that the infection rate decreases with a proper antiseptic solution (O'Grady et al, 2011; Avc1, 2015; Zhang et al, 2016; HIDER, 2019).

Antiseptic solutions frequently used for cleaning the PVC entry site in the literature are $70 \%$ Alcohol, 2\% Chlorhexidine Gluconate 70\% Alcohol (CHG), 1\% octenidine and 10\% povidone iodine (Wu et al, 2017; Karpanen et al, 2016; Dettenkofer et al, 2009; Eser Mete et al, 2009).

When antiseptics were analyzed, it was reported that maximum $2 \%$ CHG solution was the most effective antiseptic agent (Karpanen et al, 2016). In 796 patients that catheters inserted and skin antisepsis was provided by $\mathrm{CHG}$ and povidone iodine, the rate of CRI was reported as $3 \%$ in $\mathrm{CHG}$ and $10.5 \%(\mathrm{p}=0.03)$ in povidone iodine, and it was reported to be higher in povidone iodine colonization $(p=0,04)$ (Yasuda et al, 2017; Eser Mete et al, 2009).

In recent years, drawbacks are reported about the use of $\mathrm{CHG}$ in children especially babies under 2 months of age because of strong binding to skin proteins, long-lasting antimicrobial effect on skin (48 hours) and inadequate number of trials in children and infants (Serane \& Kothendaraman, 2016; O'Grady et al, 2011). Similarly, it is reported that also alcohol and povidone iodine cause many issues such as abnormalities in hormonal functions and skin problems as a result of skin absorption in children. In line with these data of these data, a safe antiseptic solution that is not harmful for childhood group is necessary.

\section{REFERENCES}

Aktaş, E., Sarı, E.N., Seremet Keskin, A., Pişkin, N., Külah, C., Cömert, F. Infection Factors and Antibiotic Susceptibilities Associated with Intravenous Catheter. Microbiology Bulletin, 45(1), 86-92, 2011.

Avc1, D. Investigation of Antibacterial Activities of Some Antiseptics and Disinfectants. Çanakkale 18 March University Health Sciences Institute, Department of Medical Microbiology, Master Thesis (Thesis Advisor Prof. Dr. Müşerref Tatman Otkun). Canakkale, Turkey, 2015.

Biçer, T. Knowledge Levels of Student Nurses Related to Intravenous Catheter Care. Istanbul Bilim University, Institute of Health Sciences, Department of Nursing, Surgical Diseases Nursing, Master Thesis (Thesis Advisor Assist. Prof. Dr Gamze Temiz). Istanbul Turkey, 2017.

Boyd, S., Aggarwal, I., Davey, P., Logan, M., Nathwani, D. Peripheral intravenous catheters: the road to quality improvement and safer patient care. Journal of Hospital Infection, 77, 37-41, 2011.

Camacho-Ortiz, A. \& Roman-Mancha, A.L. Forget skin scrubbing and other antiseptics: prevent catheter related infections using chlorhexidine plus alcohol. Ann Transl Med, 4(4), 81, 2016.

CDC. National and State HAI Progress Report: Central Line-Associated Bloodstream Infections (CLABSI)Centers for Disease Control and Prevention. Newyork, USA, 2018.

Choudhury, A., Sidjabat, H.E., Zowawi, H.M., Marsh, N., Larsen, E., Runnegar, N., Paterson, D.L., McMillan, D.J., Rickard, C.M. Skin colonization at peripheral intravenous catheter insertion sites increases the risk of catheter colonization and infection. American Journal of Infection Control, 000, 1-5, 2019.

Cihan Erdoğan, B. Phlebitis and Infiltration Development Status and Influencing Factors in Patients Undergoing Peripheral Intravenous Catheter in Neurosurgery Clinic. Adnan Menderes University, Institute of Health Sciences, Department of Nursing, Nursing Fundamentals, Master Thesis (Thesis Advisor Assist. Prof. Dr. Y1ldız Denat). Aydin, Turkey, 2014. 
Çaylan, R. Hand Hygiene. Journal of Hospital Infections, 11, 54-59, 2007.

Dettenkofer, M., Wilson, C., Gratwohl, A., Schmoor, C., Bertz, H., Frei, R., Heim, D., Luft, D., Schulz, S., Widmer, A.F. Skin disinfection with octenidine dihydrochloride for central venous catheter site care: a double-blind, randomized, controlled trial. Clin Microbiol Infect, 16, 600 606, 2009.

Eser Mete, G., Ustalar Özgen, S., Çiğdem, B., İsbir, O. Case Report: Skin Reactions Due to Povidone Iodine Skin Preparation. Faculty of Health Sciences Nursing Journal, 52-56, 2009.

Hadaway, L. Short Peripheral Intravenous Catheters and Infections. Journal of Infusion Nursing. 230240, 2012.

HIDER Turkish Hospital Infections and Control Association, National Vascular Access Management Guide,23(1), 2019.

Karagözoğlu, Ş. Nursing Care and Hot-Cold Application in Thrombophlebitis Developed as a Complication of Intravenous Fluid Therapy. CU Journal of Nursing School, 5 (1), 18-25, 2001.

Karpanen, T.J.,Casey, A.L., Whitehouse, T., Nightingale, P., Das, I., Elliott, T.S.J. Clinical evaluation of a chlorhexidine intravascular catheter gel dressing on short-term central venous catheters. American Journal of Infection Control, 44, 54-60, 2016.

Kaynar Şimşek, A. Relationship Between Duration of Peripheral Intravenous Catheters and Thrombophlebitis. Marmara University, Institute of Health Sciences, Department of Nursing Fundamentals, Master Thesis (Thesis Advisor Prof. Dr. Şule Ecevit Alpar). Istanbul Turkey, 2012

O’Grady, N.P., Alexander, M., Burns, L.A., Dellinger, E.P., Garland, J., Heard, S.O., Lipsett, P.A., Masur, H., Mermel, L.A., Pearson, M.L., Raad, I.I., Randolph, A., Rupp, M.E., Saint, S. Guidelines for the prevention of intravascular catheter-related infections, 2011. (http://www.cdc.gov/hicpac/BSI/BSI-guidelines-2011.html).

Öztürk, R. Clinic, Diagnosis and Treatment in Catheter Infections. ANKEM Journal, 14(4), 460,467, 2000 .

Özyazıcıŏlu, N., Arıkan, D. Peripheral Cannula Applications in Children. Journal of Atatürk University School of Nursing, 10 (3), 93-100, 2007.

Paşalığlu, K.B. The Effect of Catheter Stay Time on Phlebitis Development in Peripheral Intravenous Catheter Applications. Istanbul University, Institute of Health Sciences, Department of Nursing Fundamentals, Master Thesis (Thesis Advisor Assist. Prof. Hatice Kaya). Istanbul Turkey, 2012.

Pujol, M., Hornero, A., Saballs, M., Argerich, M.J., Verdahuer, R., Cisnal, M., Pena, C., Ariza, J., Gudiol, F. Clinical epidemiology and outcomes of peripheral venous catheter-related blood stream infections at a university-affiliated hospital. Journal of Hospital Infection, 67, 22-29, 2007.

Rai, C.A., Khera, C.A., Jain, M., Krishnakumar, M., Sreevastava, D.K. Bacterial colonization of peripheral intravenous cannulas in a tertiary care hospital: A cross sectional observational study. Medical Journal Armed Forces İndia, 75, 65-69, 2019

Rundjan, L. Skin antiseptic choice to reduce catheter-related blood stream infections. Paediatrica Indonesiana, 51(6), 345-350, 2011.

Serane, T., Kothendaraman, B. Incidence and Risk Factors of Infections Associated with Peripheral Intravenous Catheters. Journal of Infection Prevention, 17(3), 115-120, 2016. 
Timsit, J.F., Dubois, Y., Minet, C., Bonadona, A., Lugosi, M., Ara-Somohano, C., Hamidfar-Roy, R., Schwebel, C. New materials and devices for preventing catheter-related infections. Annals of Intensive Care, 1:34, 2-9., 2011.

Uslusoy, E. Peripheral Intravenous Catheter Applications, Phlebitis Development Status and Investigation of Affecting Factors. Dokuz Eylul University, Institute of Health Sciences, Fundamentals of Nursing, Master Thesis (Thesis Advisor Assist. Prof. Samiye Mete), Izmir. Turkey, 2006.

Wu, H., Xu, Y., Shi, J. 5\% NaHCO3 Is Appropriate for Skin Cleaning with Central Venous Catheters. The American Journal of the Medical Sciences, 353(1), 12-16., 2017.

Yasuda, H., Sanui, M., Abe, T., Shime, N., Komuro, T., Hatakeyema, J., Matsukubo, S., Kawano, S., Yamamoto, H., Andoh, K., Seo, R., Inoue, K., Noda, E., Saito, N., Nogami, S., Okamoto, K., Fuke, R., Gushima, Y., Kobayashi, A., Takebayashi, T., Lefor, A.K. Comparison of The Efficacy of Three Topical Antiseptic Solutions for the Prevention of Catheter Colonization: A Multicenter Randomized Controlled Study. Critical Care, 21:320, 1-10, 2017.

Zhang, L., Cao, S., Marsh, N., Ray-Barruel, G., Flynn, J., Larsen, E., Rickard, C.M. Infection Risks Associated with Peripheral Vascular Catheters. Journal of Infection Prevention, 17(5), 207-213., 2016. 